\title{
A Case of Torsion of a Subserosal Leiomyoma
}

\author{
Hwi-Gon Kim, M.D., Ph.D., Yong Jung Song, M.D., Ph.D., Yong Jin Na, M.D., Ph.D., \\ Ook-Hwan Choi, M.D., Ph.D. \\ Department of Obstetrics and Gynecology, Pusan National University School of Medicine, Yangsan, Korea
}

Uterine leiomyoma is one of the most common gynecological tumor, whereas acute torsion of the uterine leiomyoma is very rare. We report a case of subserosal leiomyoma that was first detected by ultrasonography, and further confirmed as torsion of subserosal leiomyoma by laparoscopic surgery. (J Menopausal Med 2013;19:147-150)

Key Words: Leiomyoma, Torsion abnormality, Uterine neoplasms

\section{Introduction}

Uterine leiomyoma is a benign subclinical gynecological tumor occurring frequently in women of childbearing age. The symptoms include menorrhagia, abnormal vaginal bleeding, dysmenorrhea, dyspareunia, pelvic pain, and urination frequency. The infrequent acute complications include thromboembolism, acute torsion, renal failure, acute pain due to secondary degeneration, mesenteric vein thrombosis, and intestinal gangrene. ${ }^{1}$ They are very rare complications that their exact incidence is not known. This paper reports the case in which the uterus was normal, but the subserosal leiomyoma was twisted to cause acute abdominal pain. Due to the rarity and difficulty of preoperative diagnosis by ultrasonography (US), computed tomography (CT) scan and magnetic resonance imaging (MRI), the diagnosis is established by operative findings. Furthermore, if the diagnosis is not made promptly, ischemic gangrene and peritonitis can occur. ${ }^{2,3}$ Thus, the torsion of subserosal leiomyoma should be diagnosed and treated by a surgical approach immediately upon suspicion. With a review of related literature, we discuss a case of torsion of subserosal leiomyoma, where the patient was misdiagnosed as secondary degeneration of uterine leiomyoma at our hospital prior to the operation.

\section{Case Report}

A 26-year-old woman visited our emergency department with acute lower abdominal pain and diarrhea on 2 October 2012. The abdominal pain developed 2 weeks ago and increased in intensity over the past few hours. She had three episodes of watery diarrhea per day. Prior to presenting to our emergency department, she received conservative treatment at a local clinic for presumptive acute gastroenteritis. Even though she was taking probiotics and analgesics, the symptoms did not improve, resulting in her referral to our emergency department. On physical examination, the temperature was $37.5^{\circ} \mathrm{C}$, and there was direct and rebound tenderness in the lower abdomen. The white blood cell count was 9,430/ $\mu \mathrm{L}$ (normal value 4,000-

Received: March 13, 2013 Revised: May 16, 2013 Accepted: May 16, 2013

Address for Correspondence: Ook-Hwan Choi, Department of Obstetrics and Gynecology, Pusan National University Yangsan Hospital, Pusan National University School of Medicine, Mulgeum-eup, Yangsan 626-770, Korea

Tel: +82-55-360-2580, Fax: +82-55-360-2160, E-mail: ohchoi@pusan.ac.kr 
$10,000 / \mu \mathrm{L}$ ) with $78.1 \%$ segment neutrophils (normal value 40.0-74.0\%), and C-reactive protein was elevated to 2.1 $\mathrm{mg} / \mathrm{dL}$ (normal value $<0.5 \mathrm{mg} / \mathrm{dL}$ ). The stool specimen was negative for white blood cells and hemoglobin. A transvaginal US showed a deviated uterus with a heterogeneous echoic globular mass, measuring $4 \times 7 \times$ $6 \mathrm{~cm}$ (Fig. 1). Pelvic MRI revealed a pelvic mass connected to the posterior aspect of the uterine corpus with a distinct border, measuring $4 \times 8.4 \times 5.5 \mathrm{~cm}$, in conjunction with a small amount of ascites. It was suspected as subserosal myoma with secondary degeneration. Others including both ovaries were unremarkable (Fig. 2). We presumed that it could be torsion or red degeneration of a pedunculated

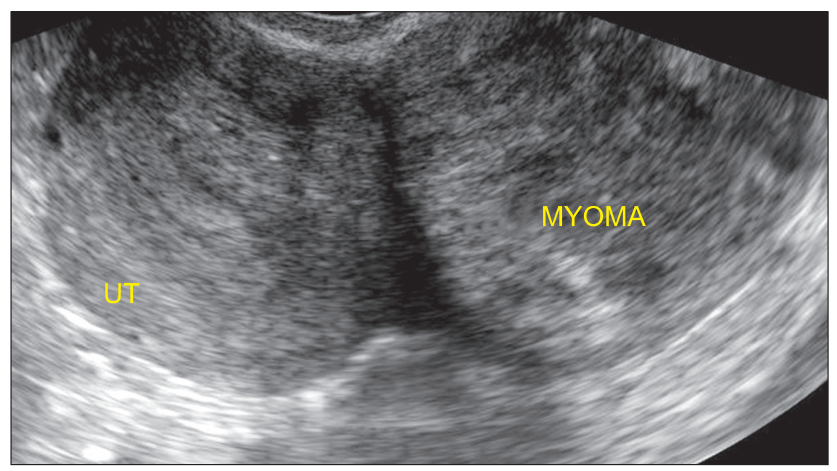

Fig. 1. Transvaginal ultrasonography shows a solid mass with heterogenous echogenicity behind the uterine corpus.

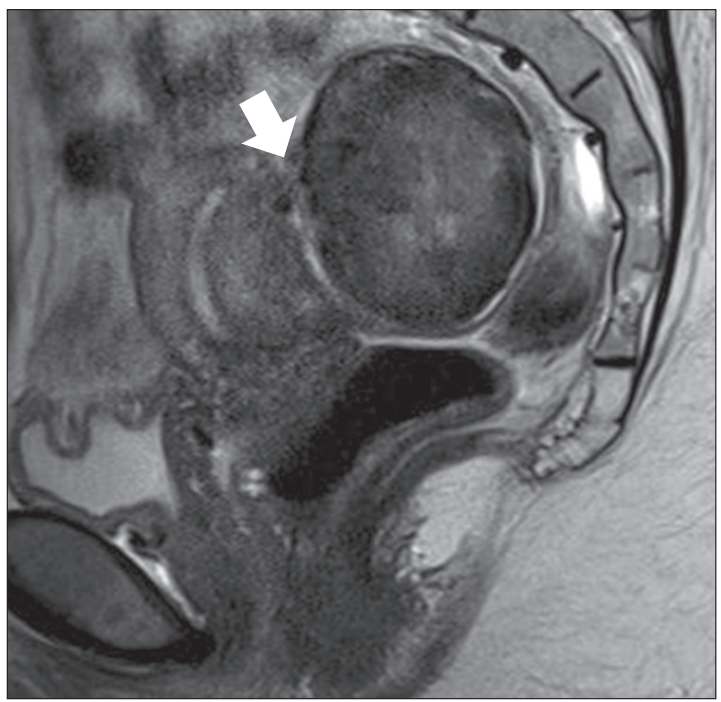

Fig. 2. Pelvic magnetic resonance imaging show moderately enhanced uterine corpus connected to a non-enhanced pelvic mass by a thin pedicle (arrow) with a small amount of ascites. subserosal uterine leiomyoma. The patient underwent an emergency diagnostic laparoscopy under general anesthesia on the day of admission. At the time of laparoscopy, a dark blue subserosal leiomyoma, approximately $8 \mathrm{~cm}$ in size, was identified on a twisted pedicle to the posterior surface of the uterus (Fig. 3). The bilateral ovaries and fallopian tubes were grossly normal and there was approximately 15 $\mathrm{mL}$ of ascites. An uncomplicated laparoscopic myomectomy was performed. On pathologic report, leiomyomatous tissue with multifocal hemorrhage and congestion was noted with dilatated vessels. Postoperative course was uneventful, and she was discharged from the hospital three days after the operation.

\section{Discussion}

The etiology of uterine leiomyoma is not yet defined, but since leiomyomas arise after menarche and decrease in size after menopause, serum estrogen concentration is expected to have influence on the physiological changes of leiomyoma. ${ }^{4}$ Recently, there has been a new approach to cause analysis, prevention and management of leiomyoma with the study of polymorphisms of estrogen receptor. ${ }^{5}$

In general, when a patient with a leiomyoma experiences acute abdominal pain, the cause may be due to other organs rather than uterine leiomyoma; therefore, it is necessary to

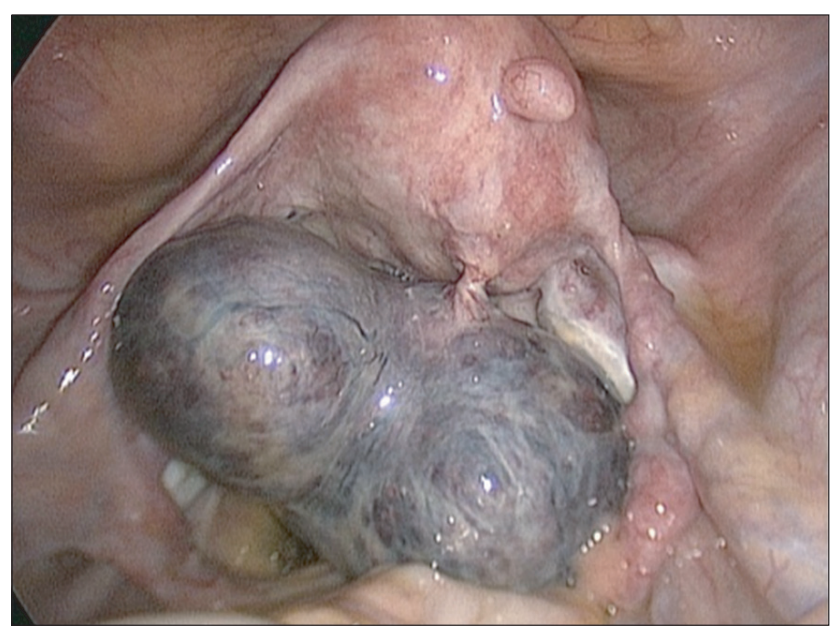

Fig. 3. A dark blue subserosal leiomyoma, approximately $8 \mathrm{~cm}$ in size, was identified on a twisted pedicle to the posterior surface of the uterus. 
exclude the possibilities of appendicitis, gastroenteritis, pelvic inflammatory disease, and torsion of ovarian cyst. Even when the acute complication is due to uterine leiomyoma, the pain is mostly caused by secondary degeneration. Although cases on torsion of subserosal leiomyoma are mostly subclinical, the presenting symptoms are non specific. In addition, due to its rarity, the diagnosis cannot be confirmed preoperatively. There have been several cases reported on torsion of uterine leiomyoma. ${ }^{6,7}$ However, there are only a handful of case reports on torsion of subserosal leiomyoma. The diagnosis is based on clinical manifestation and radiologic examination. In fact, the diagnosis is usually difficult preoperatively, partly because there are no known specific findings associated with this condition. US can identify a subserosal leiomyoma, but not its torsion. The torsion of subserosal leiomyoma may be suspected when color Doppler demonstrates twisted pedicle or when the distance between the leiomyoma and the uterus is observed. Unfortunately, these findings are not consistent across all subserosal leiomyoma. US may not be an effective method especially when twisted pedicle is thin. ${ }^{8}$ Non-necrotic or non-degenerated uterine leiomyomas on MRI show hypo-intense homogeneous T2 signal and iso-intense T1 signals. However, gangrenous uterine leiomyomas show heterogeneous hyper-intense T2 signals and hyper or iso-intense T1 signals. ${ }^{3}$ Regardless of utilization of many imaging modalities, torsion of subserosal leiomyoma is a rare condition and is seldom diagnosed preoperatively, partly because twisted pedicle is often invisibly thin and the absence of contrast enhancement is not specific.

There are nonsurgical management methods such as radiofrequency $(\mathrm{RF})$ or uterine artery embolization (UAE), but may lead to complications such as pyometra. ${ }^{9}$

For our case, the patient had suffered from abdominal pain for 2 weeks before the operation. She had been medicated at the local clinics. Because her condition had not improved, she was led to admission at our hospital. Although ultrasound and clinical findings suggested subserosal leiomyoma at our hospital, the possibility of torsion was not considered just from the US findings. We judged that the abdominal pain was caused by secondary degeneration of uterine leiomyoma, and further confirmed by laparoscopic surgery that the torsion occurred. In addition to the difficult preoperative diagnosis, a delayed diagnosis may result in ischemic necrosis and continuous abdominal pain, which in turn could lead to peritonitis. ${ }^{10}$

As discussed above, even with all the imaging modalities, it is very difficult to make an accurate preoperative diagnosis of torsion of a leiomyoma. Actually, based on our case and previous case reports, the possibility of torsion of a leiomyoma was not considered before the operation. Thus, for a patient with lower abdominal pain and tenderness around the leiomyoma, after excluding other possible causes of abdominal pain, we may consider the secondary degeneration of a uterine leiomyoma. This also applies for pregnant women, and it is a necessary step for treatment of infertility. ${ }^{11,12}$ As this case shows, for patients with persistent pain and subseroal myoma, we should consider the possibility of torsion. Furthermore, our experience suggests that laparoscopic surgery should be preferred for more accurate diagnosis and treatment. In conclusion, with a review of related literature, we report the case of a patient with torsion of a subserosal leiomyoma.

\section{References}

1. Gupta S, Manyonda IT. Acute complications of fibroids. Best Pract Res Clin Obstet Gynaecol 2009; 23: 609-17.

2. Roy C, Bierry G, El Ghali S, Buy X, Rossini A. Acute torsion of uterine leiomyoma: CT features. Abdom Imaging 2005; 30: 120-3.

3. Marcotte-Bloch C, Novellas S, Buratti MS, Caramella T, Chevallier P, Bruneton JN. Torsion of a uterine leiomyoma: MRI features. Clin Imaging 2007; 31: 360-2.

4. Choi SJ, Lee YY, Kim JH, Choi DS, Bae DS, Yoon BK. The effects of hormonal therapy on uterine leiomyomas in perimenopausal women. J Korean Soc Menopause 2004; 10: 269-75.

5. Lee SR, Hur SE, Moon HS, Chung HW. Association of polymorphisms of estrogen receptor $\alpha$ gene with the age of menopause in Korean women. J Korean Soc Menopause 2003; 9: 135-41.

6. Luk SY, Leung JL, Cheung ML, So S, Fung SH, Cheng SC. Torsion of a nongravid myomatous uterus: radiological features and literature review. Hong Kong Med J 2010; 16: 304-6.

7. Nicholson WK, Coulson CC, McCoy MC, Semelka RC. Pelvic magnetic resonance imaging in the evaluation of uterine 
torsion. Obstet Gynecol 1995; 85: 888-90.

8. Weinreb JC, Barkoff ND, Megibow A, Demopoulos R. The value of $\mathrm{MR}$ imaging in distinguishing leiomyomas from other solid pelvic masses when sonography is indeterminate. AJR Am J Roentgenol 1990; 154: 295-9.

9. Kim TH, Lee HH, Chung SH. Presenting features of pyometra including an increase in iatrogenic causes. J Low Genit Tract Dis 2011; 15: 316-7.

10. Foissac R, Sautot-Vial N, Birtwisle L, Bernard J, Fontaine A, Boujenah S, et al. Torsion of a huge pedunculated uterine leiomyoma. Am J Surg 2011; 201: e43-5.

11. Kim TH, Lee HH. How should painful cystic degeneration of myomas be managed during pregnancy? a case report and review of the literature. Iran J Reprod Med 2011; 9: 243-6.

12. Jo HH, Hwang SJ, Kim MR, Lim YT, Kim EJ, Kim JH, et al. Determinants of pregnancy rate after myomectomy in infertility women. J Korean Soc Menopause 2004; 10: 196204. 